\title{
Histone H2B lysine 120 monoubiquitination is required for embryonic stem cell differentiation
}

Cell Research (2012) 22:1402-1405. doi:10.1038/cr.2012.114; published online 31 July 2012

\section{Dear Editor,}

Histone H2B lysine 120 monoubiquitination (H2B$\mathrm{K} 120 \mathrm{ub}$ ) is a key histone modification that plays critical roles in gene transcriptional regulation and higher order chromatin organization in many species [1]. H2BK120ub has been reported to be associated with highly expressed active genes in human cells [2]. In yeast, H2B lysine 123 monoubiquitination (the homolog of mammalian H2B$\mathrm{K} 120 \mathrm{ub}$ ) is a determinant of two important histone H3 methylations, H3K4 methylation and H3K79 methylation $[1,3]$. Histone $\mathrm{H} 3 \mathrm{~K} 4 \mathrm{me} 3$ is a well-defined gene activation marker. Both H3K4 and H3K79 methylation significantly affect chromatin structure and gene transcription. In a recent study, the presence of $\mathrm{H} 2 \mathrm{~B}$ monoubiquitination was also found to stimulate $\mathrm{H} 3 \mathrm{~K} 4 \mathrm{me} 3$ during gene transcription in mammals [4], which further supports a conserved role for $\mathrm{H} 2 \mathrm{~B}$ monoubiquitination in transcription.

RAD6 is a well-characterized E2 ubiquitin-conjugating enzyme for H2B ubiquitination, and BRE1 (RNF20) is its corresponding E3 ligase [4, 5]. In our recent studies, we found that Rad6 also targets the degradation of p53 in both Drosophila and mammals [6,7]. The molecular regulation of RAD6/BRE1 and $\mathrm{H} 2 \mathrm{BK} 120 \mathrm{ub}$ has been thoroughly investigated $[5,8]$. However, the biological functions of H2BK120ub and its related enzymes in stem cell differentiation are poorly understood.

Embryonic stem cells (ESCs) have two important characteristics: self-renewal and pluripotency [9]. An increasing number of studies indicate that epigenetic regulation plays a key role in the control of these two stem cell properties [10]. The balance between histone $\mathrm{H} 3 \mathrm{~K} 4$ and $\mathrm{H} 3 \mathrm{~K} 9$ methylation is believed to be critical in determining stem cell differentiation or reprogramming [11]. Several other histone modifications, such as H3K27 methylation, also play important roles in stem cell differentiation [11]. However, are there other histone modifications that regulate stem cell differentiation? Here, we report for the first time that $\mathrm{H} 2 \mathrm{~B}$ lysine 120 monoubiquitination (H2BK120ub) is required for embryonic stem cell differentiation.

Known to be associated with the transcription of Nanog, p53 is a critical regulator of stem cell selfrenewal and reprogramming [12]. The fact that RAD6 regulates both $\mathrm{H} 2 \mathrm{BK} 120 \mathrm{ub}$ and the degradation of p53 [6, 7] raised the question of whether RAD6 also plays a role in stem cell differentiation. To test this possibility, we first performed western blot analysis to detect changes in the expression of RAD6 and several histone modifiers, including H2BK120ub. Surprisingly, we found that H2BK120ub increases substantially in the early stages of embryoid body (EB) and ESC differentiation (Figure 1A), although no or very weak RAD6 signal was detected at the same stage (Supplementary information, Figure S1 and data not shown). As shown in Figure 1A and $1 \mathrm{~B}$, there was no or very weak H2BK120ub signal in the lysates of stem cells and early differentiated ESCs, as detected by H2BK120ub-specific antibodies (data not shown, EB 0 day in Figure 1A and ES in Figure 1B). However, a dramatic increase in H2BK120ub can be detected 2 days after EB differentiation (LIF withdrawal) (LIF (-); Figure 1A) and 7 days after ESC differentiation (LIF (-); Figure 1B). H2BK120ub was stably maintained during EB differentiation (compare day 2 to 8 in LIF (-); Figure 1A), and the increase in H2BK120ub seemed to occur much earlier than the decreases in Oct4 and Nanog, indicating that the presence of H2BK120ub may be an earlier event in the initiation of stem cell differentiation. We also tested changes in $\mathrm{H} 3 \mathrm{~K} 4 \mathrm{me} 3$ levels in both differentiated EBs and ESCs, and found that H3K4me3 increased one- to twofold after 2 days of EB differentiation, although there was already a relatively high level of $\mathrm{H} 3 \mathrm{~K} 4 \mathrm{me} 3$ in ESCs (EB 0 in Figure 1A and ES in Figure 1B).

Next, we wondered whether the increase in H2B$\mathrm{K} 120 \mathrm{ub}$ was due to changes in the expression of BRE1 (RNF20) and RAD6A/B, the corresponding E3 ligase and $\mathrm{E} 2$ conjugating enzyme for catalyzing the monoubiquitination of $\mathrm{H} 2 \mathrm{~B}$ [5]. However, we found that no or very weak RAD6A/B signal can be detected during EB differentiation, indicating that RAD6A/B protein levels are 

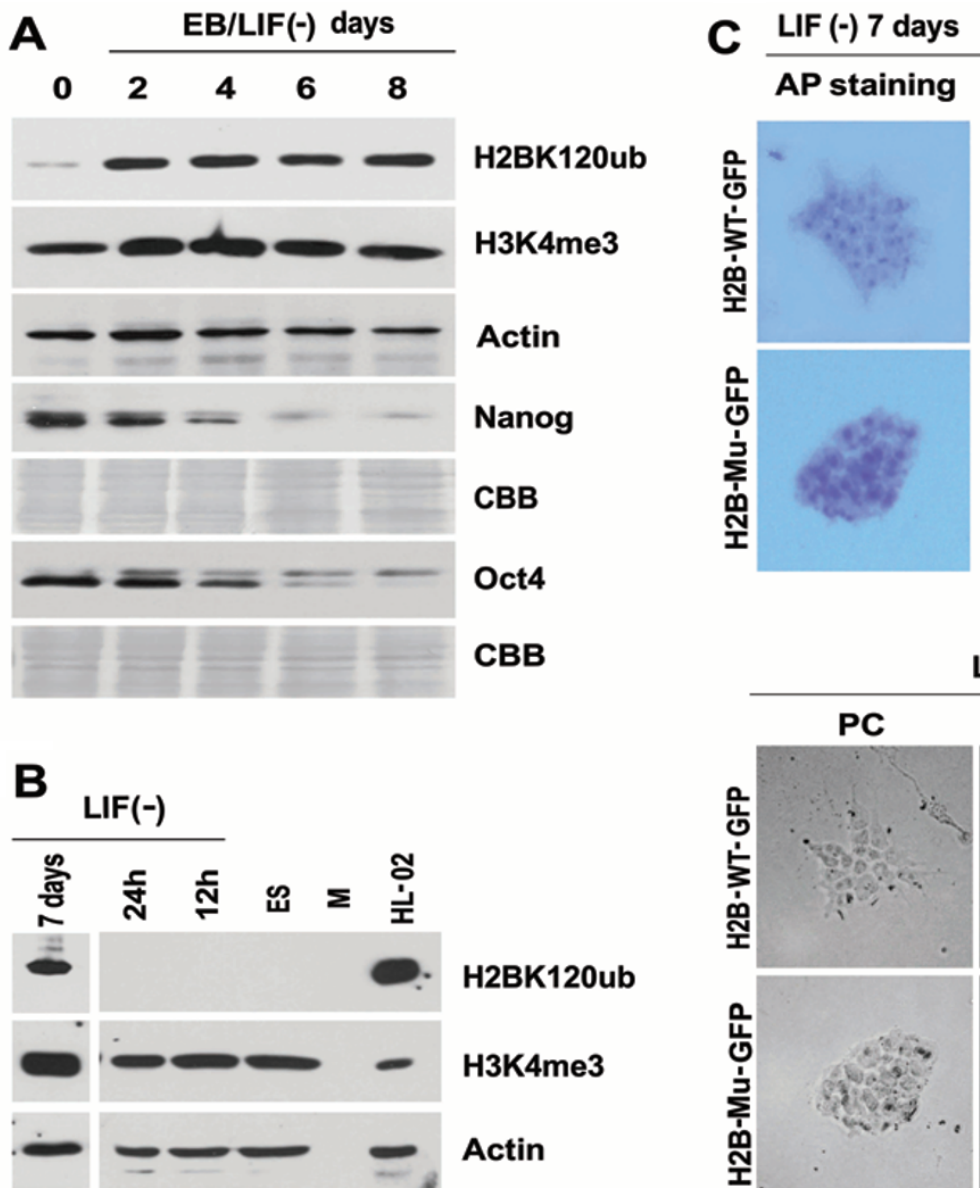

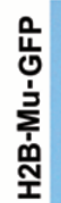
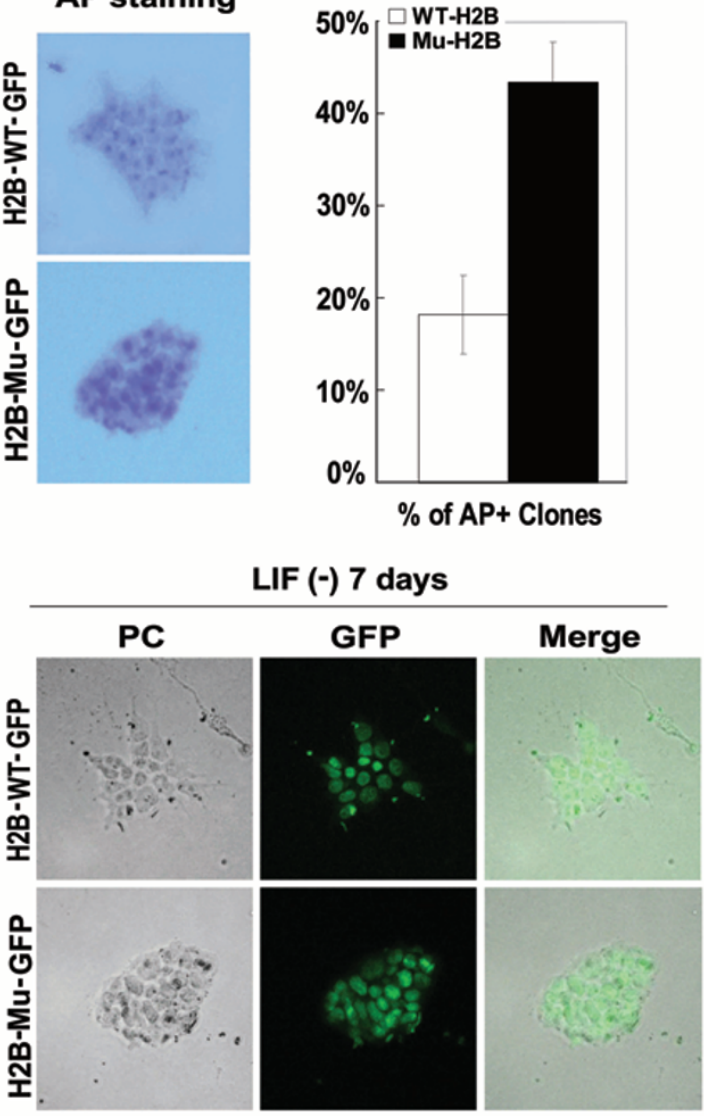

D

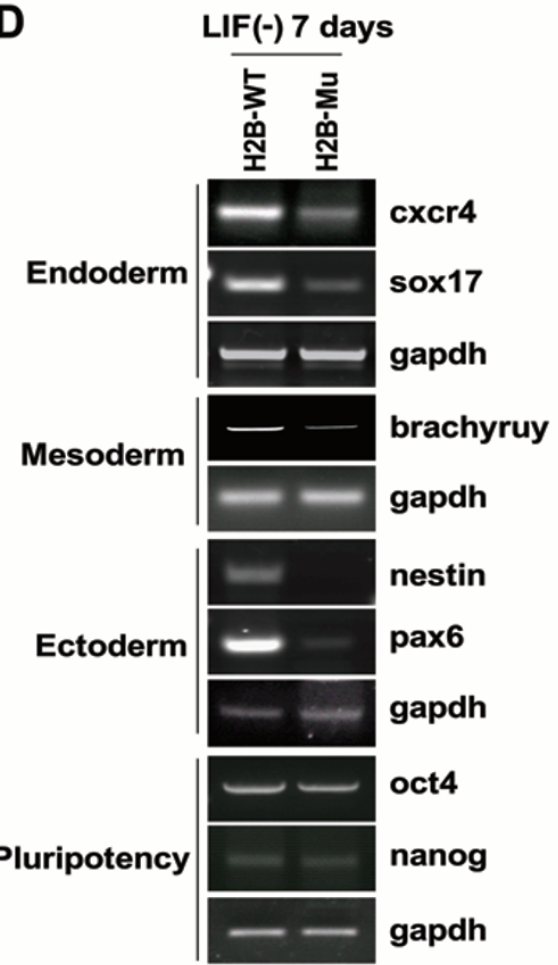

E

ChIP

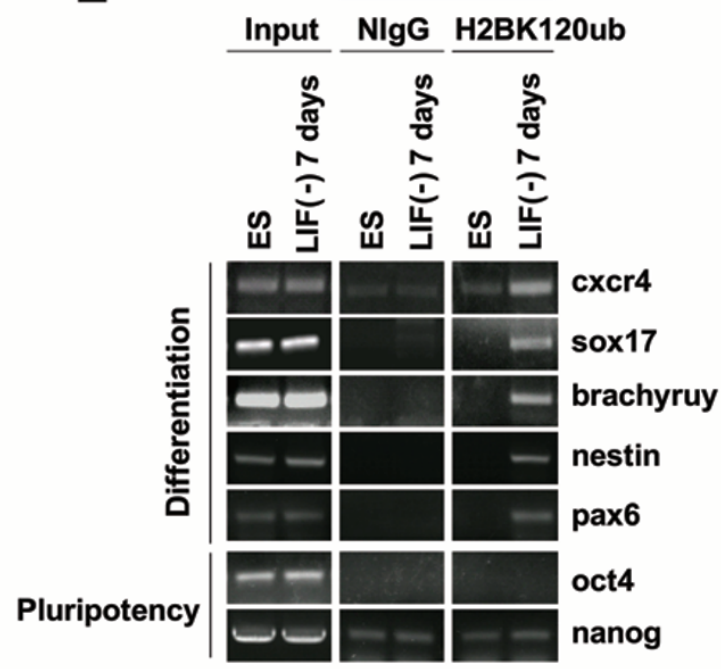

$\mathbf{F}$

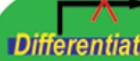

Difierentiation related genes

Pluripotent

$$
\text { ○ H2BK120ub }
$$


quite low (Supplementary information, Figure S1A and data not shown), and BRE1 protein levels decreased during EB differentiation, which is in contrast to the increase observed for H2BK120ub (Supplementary information, Figure S1A). We next tested the effect of BRE1 on the regulation of H2BK120ub in ESCs. Our results showed that knockdown of BRE1 with shRNA indeed decreases the differentiation-induced H2BK120ub level (Supplementary information, Figure S2). These data showed that BRE1 (RNF20) is the corresponding E3 ligase for H2BK120ub in ESCs, although no obvious correlation at the protein level could be found between H2BK120ub and its related enzymes (RAD6 and BRE1) during ESC differentiation. Notably, a recent report showed that the maintenance of stem cells in Drosophila seems to require a deubiquitinase, Scrawny, to maintain H2B monoubiquitination at a very low level [13], arguing the possibility that a H2BK120ub-specific deubiquitinase functions in the control of low levels of H2BK120ub in ESCs.

We next aimed to determine whether H2BK120ub directly participates in the regulation of ESC differentiation. Transfection of an H2BK120R mutant was reported to efficiently inhibit endogenous H2BK120ub levels in HEK-293T cells [2]. Therefore, we produced an H2BK120R-GFP mutant construct to test whether a reduction in H2BK120ub affects the differentiation of ESCs. H2B$\mathrm{K} 120 \mathrm{ub}$ levels in differentiated ES cells after transfection with H2B-WT-GFP (H2B-WT) or H2BK120R-GFP (H2B-Mu) were determined. The results showed that the H2BK120R mutant construct indeed resulted in efficient downregulation of H2BK120ub in differentiated ESCs (7 days after LIF withdrawal) (Supplementary information, Figure S3). ES cells transfected with H2B-WT or H2B$\mathrm{Mu}$ were further cultured under LIF withdrawal (LIF (-)) conditions for 7 days to induce differentiation followed by alkaline phosphatase (AP) staining to examine the self-renewal property of ES cells. Alkaline phosphatase is a stem cell membrane marker, and elevated expression of this enzyme is associated with undifferentiated pluripotent stem cells. All primate pluripotent stem cells, such as embryonic stem (ES), embryonic germ (EG) and embryonal carcinoma (EC) cells, show alkaline phosphatase activity, while differentiated cells have lower AP activity. The results showed that H2B-Mu ES cells have much more intense AP staining compared to that in H2BWT ES cells (43\% AP-positive colonies in H2B-Mu and $18 \%$ in $\mathrm{H} 2 \mathrm{~B}-\mathrm{WT}$ ) (Figure 1C, upper panel), indicating less cell differentiation. In addition, H2B-WT and H2B$\mathrm{Mu}$ ES cell colonies have quite different morphology. $\mathrm{H} 2 \mathrm{~B}-\mathrm{Mu}$ clones seem to have a better pluripotency morphology than H2B-WT (Figure 1C, lower panel). We also tested the effect of BRE1 (RNF20) on ESC differentiation using a similar method (AP staining). Our results showed that knockdown of BRE1 with shRNA inhibits ESC differentiation ability (37\% AP-positive colonies in shRNF20 and 19\% in shCont) (Supplementary information, Figure S4A), and decreases the expression of differentiation-related genes (Supplementary information, Figure S4B).

To further elucidate the role of H2BK120ub in regulating stem cell differentiation, we next performed RTPCR using the H2B-WT and H2B-Mu strains differentiated for 7 days (LIF (-)) to detect changes in expression of marker genes associated with ESCs and differentiated ESCs. From the results shown in Figure 1D, we observe that the presence of the H2BK120R mutant inhibits the transcription of differentiation-related genes after LIF withdrawal for 7 days (differentiation status), while the mutant has little effect on the expression of pluripotencyrelated genes. This indicated that the presence of $\mathrm{H} 2 \mathrm{~B}-$

Figure $1 \mathrm{H} 2 \mathrm{BK} 120 \mathrm{ub}$ determines mouse embryonic stem cell differentiation. (A) The level of H2BK120ub increases significantly after embryoid body (EB) differentiation. EBs were prepared and differentiated for the indicated number of days in medium without LIF $(\operatorname{LIF}(-))$. EBs were lysed and western blots were performed using the indicated antibodies. CBB indicates Coomassie Brilliant Blue staining. (B) H2BK120ub was undetectable in ES cells, but a striking increase was observed after ESC differentiation. ESCs were cultured without LIF $(\operatorname{LIF}(-))$ for varying times and lysed for western blot assays with the indicated antibodies. H3K4 trimethylation (H3K4me3) showed no obvious changes during the first $24 \mathrm{~h}$ of differentiation. $\mathrm{M}$ indicates marker lane; HL-02 was used as a positive control for antibody activity. (C) H2BK120 mutation inhibits the efficiency of ESC differentiation. AP staining of H2B-WT and H2B-Mu mutant ESCs was performed after ESCs were cultured without LIF $(\mathrm{LIF}(-))$ for 7 days (upper panel). The percentage of AP stained clones is shown on the bar diagram. The morphology of H2BWT and H2B-Mu mutant ESCs after being cultured without LIF (LIF(-)) for 7 days is shown (lower panel). (D) The H2BK120R mutant inhibits the transcription of differentiation-related genes during ESC differentiation. RT-PCR assays were performed after H2B-WT and H2B-Mu mutant ESCs were cultured without LIF for 7 days. (E) H2BK120ub is preferentially associated with differentiation-related genes. ChIP analysis was performed using anti-H2BK120ub antibodies in H2B-WT and H2B-Mu mutant ESCs cultured without LIF for 7 days. NlgG was used as a negative control for the antibody. (F) A working model for $\mathrm{H} 2 \mathrm{BK} 120 \mathrm{ub}$ in the regulation of ESC differentiation. The presence of H2BK120ub in the regulatory elements of differentiationrelated genes triggers changes in transcription and subsequent ESC differentiation. 
K120ub is essential for the transcription of differentiation-related genes but not pluripotency-related genes.

Next, we performed chromatin immunoprecipitation (ChIP) analysis to determine the direct role of H2B$\mathrm{K} 120 \mathrm{ub}$ in the transcription of the tested genes during ESC differentiation. As shown in Figure 1E, we found that $\mathrm{H} 2 \mathrm{BK} 120 \mathrm{ub}$ is preferentially enriched in the coding region of differentiation-related genes but not pluripotency-related genes after ESC differentiation. These results confirmed that the presence of $\mathrm{H} 2 \mathrm{BK} 120 \mathrm{ub}$ is associated with differentiation-related genes, and the reduction in H2BK120ub affected their transcription, triggering further alterations in ESC differentiation.

In summary, we report a novel role for H2BK120ub in ESC differentiation. We further propose that H2BK120ub may be a key histone modification in triggering ESC differentiation. Further studies are necessary to elucidate its regulatory mechanism and the enzymes responsible for this modification. Materials and Methods are described in the Supplementary information, Data S1. Primers used in this study are shown in Supplementary information, Table S1.

During the revision of our manuscript, two reports were published in $\mathrm{Mol}$ Cell $[14,15]$. They show similar results to our data and support our conclusion significantly.

\section{Acknowledgments}

This work was supported by the National Basic Research Program of China (973 Program) from the Ministry of Science and Technology (2011CB965300, 2009CB825603).

$$
\begin{aligned}
& \text { Su Chen }{ }^{1,2,3,{ }^{*}} \text {, Juan } \mathrm{Li}^{1,2,{ }^{*}} \text {, Da-Liang Wang }{ }^{3, *} \text {, } \\
& \text { Fang-Lin Sun }{ }^{1,2,3}
\end{aligned}
$$

${ }^{I}$ School of Life Sciences and Technology, Tongji University, Shanghai 200092, China; ${ }^{2}$ The Advanced Institute of Translational Medicine, Tongji University, Shanghai 200092, China; ${ }^{3}$ Institute of Epigenetics and Cancer Research, School of Medicine, Tsinghua University, Beijing 100084, China

*These three authors contributed equally to this work. Correspondence: Fang-Lin Sun

Tel: +86-21-65980910

E-mail: sfl@tongji.edu.cn

\section{References}

1 Sun ZW, Allis CD. Ubiquitination of histone H2B regulates H3 methylation and gene silencing in yeast. Nature 2002; 418:104-108.

2 Minsky N, Shema E, Field Y, et al. Monoubiquitinated H2B is associated with the transcribed region of highly expressed genes in human cells. Nat Cell Biol 2008; 10:483-488.

3 Nakanishi S, Lee JS, Gardner KE, et al. Histone H2BK123 monoubiquitination is the critical determinant for $\mathrm{H} 3 \mathrm{~K} 4$ and H3K79 trimethylation by COMPASS and Dot1. J Cell Biol 2009; 186:371-377.

4 Kim J, Guermah M, McGinty RK, et al. RAD6-Mediated transcription-coupled $\mathrm{H} 2 \mathrm{~B}$ ubiquitylation directly stimulates H3K4 methylation in human cells. Cell 2009; 137:459-471.

5 Kim J, Hake SB, Roeder RG. The human homolog of yeast BRE1 functions as a transcriptional coactivator through direct activator interactions. Mol Cell 2005; 20:759-770.

6 Chen S, Wei HM, Lv WW, Wang DL, Sun FL. E2 ligase dRad6 regulates DMP53 turnover in Drosophila. J Biol Chem 2011; 286:9020-9030.

7 Chen S, Wang DL, Liu Y, Zhao L, Sun FL. RAD6 regulates the dosage of p53 by a combination of transcriptional and posttranscriptional mechanisms. Mol Cell Biol 2012; 32:576587.

8 Kim J, Roeder RG. Direct Bre1-Pafl complex interactions and RING finger-independent Bre1-Rad6 interactions mediate histone H2B ubiquitylation in yeast. J Biol Chem 2009; 284:20582-20592.

9 Zipori D. The stem state: plasticity is essential, whereas selfrenewal and hierarchy are optional. Stem Cells 2005; 23:719726.

10 Orkin SH, Hochedlinger K. Chromatin connections to pluripotency and cellular reprogramming. Cell 2011; 145:835-850.

11 Jiang H, Shukla A, Wang X, et al. Role for Dpy-30 in ES cellfate specification by regulation of $\mathrm{H} 3 \mathrm{~K} 4$ methylation within bivalent domains. Cell 2011; 144:513-525.

12 Lin T, Chao C, Saito S, et al. p53 induces differentiation of mouse embryonic stem cells by suppressing Nanog expression. Nat Cell Biol 2005; 7:165-171.

13 Buszczak M, Paterno S, Spradling AC. Drosophila stem cells share a common requirement for the histone H2B ubiquitin protease scrawny. Science 2009; 323:248-251.

14 Fuchs G, Shema E, Vesterman R, et al. RNF20 and USP44 regulate stem cell differentiation by modulating $\mathrm{H} 2 \mathrm{~B}$ monoubiquitylation. Mol Cell 2012; 46:662-673.

15 Karpiuk O, Najafova Z, Kramer F, et al. The histone H2B monoubiquitination regulatory pathway is required for differentiation of multipotent stem cells. Mol Cell 2012; 46:705713.

(Supplementary information is linked to the online version of the paper on the Cell Research website.) 